\title{
EFEITO DE REGULADORES DE CRESCIMENTO EM UVA APIRÊNICA, CV. BRS CLARA ${ }^{1}$
}

\author{
JAIR COSTA NACHTIGAL², UMBERTO ALMEIDA CAMARGO², JOÃO DIMAS GARCIA MAIA ${ }^{3}$
}

\begin{abstract}
RESUMO - O presente trabalho teve por objetivo verificar o efeito da aplicação de diferentes concentrações do ácido giberélico ( $\mathrm{AG}_{3}$ ), do thidiazuron (TDZ) e do forchlorfenuron (CPPU) no aumento do tamanho dos cachos e das bagas e no teor de sólidos solúveis totais de uva sem semente, cv. BRS Clara. As plantas foram conduzidas no sistema de latada, sobre o porta-enxerto IAC 572, no espaçamento 2,5 x $2,0 \mathrm{~m}$ e com irrigação por microaspersão. Os experimentos foram conduzidos na Estação Experimental de Viticultura Tropical, da Embrapa Uva e Vinho, em Jales-SP. Os reguladores de crescimento foram aplicados via pulverização localizada no cacho, utilizando-se de concentrações de 0 a $4 \mathrm{mg} . \mathrm{L}^{-1}$ de CPPU; 0 a $10 \mathrm{mg} . \mathrm{L}^{-1}$ de TDZ, e de 0 a $90 \mathrm{mg} . \mathrm{L}^{-1}$ de $\mathrm{AG}_{3}$, isolados ou em conjunto. De modo geral, a aplicação dos reguladores de crescimento promove a melhoria da qualidade dos cachos da cv. BRS Clara; o uso do TDZ e do CPPU em conjunto com o $\mathrm{AG}_{3}$ produz um efeito sinérgico, proporcionando melhor resposta do que o uso isolado do $\mathrm{AG}_{3}$; os tratamentos com $60 \mathrm{mg} . \mathrm{L}^{-1}$ de $\mathrm{AG}_{3} ; 20 \mathrm{mg} . \mathrm{L}^{-1}$ de $\mathrm{AG}_{3}+4 \mathrm{mg} . \mathrm{L}^{-1}$ de CPPU, e $10 \mathrm{mg} . \mathrm{L}^{-1}$ de AG 3 + $5 \mathrm{mg} . \mathrm{L}^{-1}$ de TDZ proporcionam os melhores resultados para o aumento do diâmetro das bagas; a aplicação de $\mathrm{AG}_{3}$ antes da floração da cv. BRS Clara provoca abortamento excessivo, reduzindo a qualidade comercial dos cachos; a utilização de concentrações elevadas dos reguladores reduz o teor de sólidos solúveis totais.
\end{abstract}

Termos de indexação: Vitis sp., uvas de mesa, ácido giberélico, thidiazuron, forchlorfenuron.

\section{EFFECT OF GROWTH REGULATORS ON THE SEEDLESS GRAPE CV. BRS CLARA}

\begin{abstract}
The objective of this study was to evaluate the effect of different concentrations of gibberellic acid (AG ( $_{3}$, thidiazuron (TDZ) and forchlorfenuron (CPPU) on the size of bunches and berries and on the total soluble solids content, when applied in different concentrations on the seedless grapes, cv. BRS Clara. The grapevines were grafted on the rootstock IAC 572, trained in the pergola system and, spaced $2.5 \times 2.0 \mathrm{~m}$ and irrigated by micro sprinklers. The experiments were carried out at the Embrapa Grape and Wine Experimental Station for Tropical Viticulture in Jales, SP. The bunches were directly sprayed with the plant growth regulators, either individually or combined, in concentrations from 0 to $4 \mathrm{mg}$. $\mathrm{L}^{-1}$ of CPPU; 0 to $10 \mathrm{mg} . \mathrm{L}^{-1}$ of TDZ and 0 to $90 \mathrm{mg} . \mathrm{L}^{-1}$ of $\mathrm{AG}_{3}$. Generally, the application of growth regulators improved the quality of the bunches of the cv. BRS Clara; the use of TDZ and CPPU, combined with $\mathrm{AG}_{3}$, produce a synergistic effect, causing a better response than the isolated use of $\mathrm{AG}_{3}$; the treatments with $60 \mathrm{mg} . \mathrm{L}^{-1}$ of $\mathrm{AG}_{3}, 20 \mathrm{mg} . \mathrm{L}^{-1}$ of $\mathrm{AG}_{3}+4 \mathrm{mg} . \mathrm{L}^{-1}$ of CPPU and $10 \mathrm{mg} . \mathrm{L}^{-1}$ of $\mathrm{AG}_{3}+5 \mathrm{mg}$. $\mathrm{L}^{-1}$ of TDZ provide the best results for increase in the berries diameter; the application of $\mathrm{AG}_{3}$ before flowering cause excessive abortion which reduced the commercial value of the bunches. High concentrations of plant growth regulators decrease the content of total soluble solids.
\end{abstract}

Index terms: Vitis sp., table grape, gibberellic acid, thidiazuron, forchlorfenuron.

A produção de uvas finas de mesa no Brasil está concentrada, principalmente, nas regiões norte do Paraná, noroeste de São Paulo, Submédio São Francisco e Pirapora-MG, sendo que a cultivar Itália e suas mutações representam cerca de $90 \%$ da produção (Nachtigal, 2003). Entretanto, segundo Protas et al. (2002), é necessário que ocorra a conversão urgente da matriz produtiva de uvas finas de mesa em favor da produção de uvas sem sementes (apirênicas), como forma de habilitar a viticultura brasileira a competir interna e externamente neste mercado, já que estas apresentam melhor aceitação pelos consumidores.

Várias tentativas já foram realizadas por empresas e produtores no sentido de viabilizar o cultivo das principais cultivares de uvas sem sementes, como Thompson Seedless, Crimson Seedless e Superior Seedless, nas condições brasileiras. Entretanto, tais cultivares apresentam sérios problemas de adaptação e fertilidade baixa, produzindo colheitas irregulares, o que, algumas vezes, as inviabiliza comercialmente (Camargo et al., 1997; Leão, 2001; Camargo, 2003).

Desde 1997, a Embrapa Uva e Vinho vem desenvolvendo o programa de melhoramento genético para criação de cultivares de uvas de mesa sem sementes adaptadas às regiões produtoras do País e com qualidade para competir no mercado externo. Em 2003, foram lançadas as primeiras cultivares: BRS Morena, BRS Linda e BRS Clara. A BRS Clara, obtida do cruzamento entre CNPUV 154-147 x Centennial Seedless, é uma cultivar produtiva (cerca de $30 \mathrm{t}^{\mathrm{h}} \mathrm{ha}^{-1}$. ano ${ }^{-1}$ ), que apresenta cacho de tamanho médio a grande; baga elíptica, verde-amarelada, chegando a amarela quando exposta ao sol; polpa incolor e firme; sabor moscatel leve e agradável, e traço de semente grande, porém imperceptível à mastigação (Camargo et al., 2003).

Em conjunto com o programa de melhoramento, são desenvolvidos trabalhos que permitem os ajustes de manejo no sentido de adequar o material às exigências do mercado, principalmente com melhorias das características dos cachos e das bagas.

A cultivar BRS Clara apresenta, naturalmente, bagas com cerca $15 \mathrm{~mm}$ de diâmetro e $20 \mathrm{~mm}$ de comprimento (Camargo et al., 2003). Entretanto, a comercialização de uvas de mesa depende da aparência do produto, especialmente do tamanho das bagas (Reynolds et al., 1992). Nesse sentido, a aplicação exógena de reguladores de crescimento pode contribuir para a melhoria da qualidade do cacho e facilitar a comercialização dessa e de outras cultivares de uvas sem sementes (Nachtigal, 2003). Dentre os reguladores de crescimento mais utilizados para esta finalidade, estão o ácido giberélico $\left(\mathrm{AG}_{3}\right)$, o forchlorfenuron (CPPU), o quinmerac (IUPAC), o thidiazuron (TDZ) e a benzilaminopurina (BAP) (Pires \& Botelho, 2002).

Dependendo da época e da dose, os reguladores de crescimento podem ser utilizados com diferentes finalidades. O ácido giberélico, por exemplo, quando aplicado em plena floração, pode promover menor pegamento de flores e alongamento da ráquis, o que torna os cachos mais soltos. Já, quando aplicado cerca de 15 dias após o florescimento ou quando as bagas estiverem com 3 a $5 \mathrm{~mm}$ de diâmetro, promove aumento do tamanho das bagas (Pires \& Botelho, 2001).

O presente trabalho teve por objetivo verificar o efeito da aplicação do $\mathrm{AG}_{3}$, do TDZ e do CPPU, em diferentes concentrações, no aumento do tamanho dos cachos e das bagas e no teor de sólidos solúveis totais de uva sem semente, cv. BRS Clara.

Os experimentos foram realizados em plantas da cv. BRS Clara enxertadas no ano de 2000, sobre o porta-enxerto IAC 572 'Jales', no espaçamento $2,5 \times 2,0 \mathrm{~m}$, conduzidas no sistema de latada e com irrigação por microaspersão, sendo conduzidos durante os anos de 2001 e de 2003, na Estação Experimental de Viticultura Tropical, da Embrapa Uva

\footnotetext{
1 (Trabalho 003/2005). Recebido: 05/01/2005. Aceito para publicação: 19/07/2005. Apoio MAPA/Profruta/CNPq.

${ }^{2}$ Embrapa Uva e Vinho, Caixa Postal 130, CEP: 95700-000, Bento Gonçalves-RS, Brasil. E-mail: jair@cnpuv.embrapa.br

${ }^{3}$ Embrapa Uva e Vinho/Estação Experimental de Viticultura Tropical, Caixa Postal 241, CEP: 15700-000, Jales-SP, Brasil.
} 
e Vinho, em Jales, região noroeste do Estado de São Paulo.

No ano de 2001, a poda de produção das plantas foi realizada em 10-07, e os tratamentos com $\mathrm{AG}_{3}$, nas concentrações de $0 ; 30 ; 60$ e 90mg. $\mathrm{L}^{-1}$, foram realizados em 06-09, quando as bagas apresentavam de 6 a $8 \mathrm{~mm}$ de diâmetro, sendo repetidos cinco dias após. No ano de 2003, a poda de produção foi realizada em 18-03 e foram conduzidos três experimentos distintos: um, testando-se as concentrações de $0 ; 20$ e $40 \mathrm{mg} . \mathrm{L}^{-1} \mathrm{de} \mathrm{AG}_{3}$, aplicadas antes (14-04) e depois da floração (02-05), quando as bagas apresentavam de 3 a $5 \mathrm{~mm}$ de diâmetro, e outros dois experimentos utilizando-se de concentrações $0 ; 2$ e $4 \mathrm{mg} . \mathrm{L}^{-1}$ de CPPU; 0 ; 5 e $10 \mathrm{mg} . \mathrm{L}^{-1} \mathrm{de}$ TDZ, ambas combinadas com $0 ; 10$ e $20 \mathrm{mg} . \mathrm{L}^{-1}$ de $\mathrm{AG}_{3} \mathrm{e}$ aplicados em 07-05, quando as bagas apresentavam de 5 a $7 \mathrm{~mm}$ de diâmetro.

Em todos os experimentos, as aplicações dos reguladores foram feitas via pulverização localizada no cacho, até o ponto de escorrimento, utilizando-se de espalhante adesivo $(0,001 \%)$. Durante a condução dos experimentos, foram feitas as operações de manejo, controle fitossanitário, irrigações, adubações e outras, conforme as exigências da cultura.

As avaliações foram feitas por ocasião da maturação das uvas, considerando-se as massas frescas do cacho, do engaço e da baga, determinadas por meio de balança analítica; o comprimento e o diâmetro médio das bagas, utilizando-se de paquímetro; e o teor de sólidos solúveis totais (SST), por meio de refratômetro manual.

Os experimentos foram conduzidos no delineamento experimental inteiramente casualizado, utilizando-se de um cacho por repetição e 10 repetições no ano de 2001 e 6 repetições no ano de 2003. Os dados foram submetidos à análise de variância e, para a comparação das médias dos tratamentos, foi utilizado o teste de Tukey, ao nível de $5 \%$ de probabilidade.

Os resultados relativos ao efeito dos reguladores de crescimento na melhoria da qualidade de cachos da cv. BRS Clara são apresentados nas Tabelas 1 a 4 .

Com relação à massa fresca do cacho, os melhores resultados foram observados nos tratamentos com doses mais elevadas de $\mathrm{AG}_{3} \mathrm{e}$ com duas aplicações $\left(90+60 \mathrm{mg}\right.$. $\mathrm{L}^{-1}$ e $90+90 \mathrm{mg}$. $\left.\mathrm{L}^{-1}\right)$, com 632,58 e $630,31 \mathrm{~g}$, respectivamente, diferindo estatisticamente somente do tratamento sem aplicação, cujos cachos apresentaram, em média, $398,94 \mathrm{~g}$ de peso (Tabela 1). O aumento na massa dos cachos deveuse, provavelmente, ao aumento no tamanho das bagas, uma vez que o $\mathrm{AG}_{3}$, quando aplicado na fase de chumbinho, não tem efeito sobre o número de bagas.

A utilização do ácido giberélico em conjunto com o CPPU (Tabela 2) e com o TDZ (Tabela 3) não produziu efeito estatístico significativo, embora tenha havido aumento da massa fresca dos cachos com o aumento das concentrações dos reguladores de crescimento. Pelos dados apresentados na Tabela 4, ficou claro o efeito deletério da aplicação do $\mathrm{AG}_{3}$ antes do florescimento nessa cultivar, ocasionando elevado índice de abortamento das flores e variabilidade na massa fresca dos cachos, o que comprometeu a qualidade comercial.

TABELA 1 - Influência do ácido giberélico, aplicado nas fases de chumbinho e 5 dias após, sobre a massa fresca do cachos, do engaço e das bagas, comprimento e diâmetro das bagas e teor de sólidos solúveis totais da cv. BRS Clara. Jales, 2001.

\begin{tabular}{|c|c|c|c|c|c|c|}
\hline \multirow{3}{*}{$\begin{array}{c}\text { Tratamento } \\
\mathrm{AG}_{\mathbf{3}} \\
\left(\mathrm{mg} \cdot \mathrm{L}^{-1}\right)\end{array}$} & \multicolumn{3}{|c|}{ Massa Fresca } & \multirow[t]{2}{*}{ Comprimento Baga } & \multirow[t]{2}{*}{ Diâmetro Baga } & \multirow{3}{*}{$\begin{array}{c}\text { SST } \\
\left({ }^{0} \text { Brix }\right)\end{array}$} \\
\hline & Cacho & Engaço & Baga & & & \\
\hline & & (g) & & \multicolumn{2}{|c|}{$(\mathbf{m m})$} & \\
\hline $0+0$ & $398,94 b$ & $11,24 \mathrm{ab}$ & $2,61 \mathrm{c}$ & $19,89 \mathrm{c}$ & $15,09 \mathrm{a}$ & $22,30 \mathrm{a}$ \\
\hline $0+30$ & $410,04 \mathrm{ab}$ & $8,48 b$ & $2,93 \mathrm{abc}$ & $20,20 b c$ & $15,70 \mathrm{a}$ & $22,27 \mathrm{a}$ \\
\hline $0+60$ & $430,83 \mathrm{ab}$ & $11,57 \mathrm{ab}$ & $2,81 \mathrm{bc}$ & $20,29 b c$ & $15,48 \mathrm{a}$ & $21,35 \mathrm{ab}$ \\
\hline $0+90$ & $477,39 \mathrm{ab}$ & $12,69 \mathrm{ab}$ & $4,04 \mathrm{a}$ & $21,06 \mathrm{abc}$ & $15,71 \mathrm{a}$ & $19,64 \mathrm{bcdef}$ \\
\hline $30+0$ & $485,79 \mathrm{ab}$ & $13,13 \mathrm{ab}$ & $3,04 \mathrm{abc}$ & $21,20 \mathrm{abc}$ & $15,89 \mathrm{a}$ & $20,69 \mathrm{abc}$ \\
\hline $30+30$ & $545,88 \mathrm{ab}$ & $14,77 \mathrm{ab}$ & $3,53 \mathrm{abc}$ & $21,60 \mathrm{abc}$ & $16,39 a$ & $20,10 \mathrm{bcd}$ \\
\hline $30+60$ & $587,56 \mathrm{ab}$ & $16,48 \mathrm{a}$ & $2,99 \mathrm{abc}$ & $20,98 \mathrm{abc}$ & $15,85 \mathrm{a}$ & $19,46 \mathrm{bcdef}$ \\
\hline $30+90$ & $464,58 \mathrm{ab}$ & $12,52 \mathrm{ab}$ & $3,83 \mathrm{ab}$ & $21,85 \mathrm{ab}$ & $16,51 \mathrm{a}$ & $18,48 \mathrm{def}$ \\
\hline $60+0$ & $511,22 \mathrm{ab}$ & $12,57 \mathrm{ab}$ & $3,34 \mathrm{abc}$ & $21,79 \mathrm{ab}$ & $16,31 \mathrm{a}$ & $20,26 a b c d$ \\
\hline $60+30$ & $528,69 \mathrm{ab}$ & $14,47 \mathrm{ab}$ & $3,25 \mathrm{abc}$ & $21,71 \mathrm{ab}$ & $16,15 \mathrm{a}$ & $19,16 \mathrm{cdef}$ \\
\hline $60+60$ & $483,91 \mathrm{ab}$ & $12,27 \mathrm{ab}$ & $3,27 \mathrm{abc}$ & $21,39 \mathrm{abc}$ & $16,46 a$ & $19,18 \mathrm{cdef}$ \\
\hline $60+90$ & $550,30 \mathrm{ab}$ & $14,26 a b$ & $3,39 \mathrm{abc}$ & $22,13 \mathrm{a}$ & $16,47 \mathrm{a}$ & $17,78 \mathrm{ef}$ \\
\hline $90+0$ & $541,45 \mathrm{ab}$ & $13,89 \mathrm{ab}$ & $3,19 \mathrm{abc}$ & $21,07 \mathrm{abc}$ & $16,02 \mathrm{a}$ & 19,84 bcde \\
\hline $90+30$ & $600,63 \mathrm{ab}$ & $16,28 \mathrm{ab}$ & $3,36 a b c$ & $21,44 a b c$ & $16,14 \mathrm{a}$ & $18,98 \mathrm{cdef}$ \\
\hline $90+60$ & $632,58 \mathrm{a}$ & $16,66 \mathrm{a}$ & $3,37 \mathrm{abc}$ & $21,69 \mathrm{ab}$ & $16,20 \mathrm{a}$ & $18,78 \mathrm{cdef}$ \\
\hline $90+90$ & $630,31 \mathrm{a}$ & $17,37 \mathrm{a}$ & $3,37 \mathrm{abc}$ & $22,21 \mathrm{a}$ & $16,58 \mathrm{a}$ & $17,56 \mathrm{f}$ \\
\hline CV (\%) & 28,02 & 36,37 & 21,18 & 5,39 & 6,17 & 6,82 \\
\hline
\end{tabular}

Médias seguidas por letras distintas, dentro da mesma coluna, diferem entre si, pelo teste de Tukey, ao nível de 5\% de probabilidade.

TABELA 2 - Influência do ácido giberélico e do forchlorfenuron sobre a massa fresca do cachos, do engaço e das bagas, comprimento e diâmetro das bagas e teor de sólidos solúveis totais da cv. BRS Clara. Jales, 2003.

\begin{tabular}{|c|c|c|c|c|c|c|}
\hline \multirow{3}{*}{$\begin{array}{c}\text { Tratamento } \\
\left(\mathrm{AG}_{3}+\mathrm{CPPU}\right) \\
\left(\mathrm{mg}^{\left.-\mathrm{L}^{-1}\right)}\right.\end{array}$} & \multicolumn{3}{|c|}{ Massa Fresca } & \multirow[t]{2}{*}{ Comprimento Baga } & \multirow[t]{2}{*}{ Diâmetro Baga } & \multirow{3}{*}{$\begin{array}{c}\text { SST } \\
\left({ }^{0} \text { Brix }\right)\end{array}$} \\
\hline & Cacho & Engaço & Baga & & & \\
\hline & \multicolumn{3}{|c|}{ (g) } & \multicolumn{2}{|c|}{$(\mathbf{m m})$} & \\
\hline $0+0$ & $612,14 a$ & $12,30 \mathrm{a}$ & $3,00 \mathrm{a}$ & $19,86 a$ & $15,30 \mathrm{~b}$ & $21,95 \mathrm{a}$ \\
\hline $5+2$ & $788,68 \mathrm{a}$ & $15,88 \mathrm{a}$ & $3,61 \mathrm{ab}$ & $21,42 \mathrm{a}$ & $16,32 \mathrm{ab}$ & $20,60 \mathrm{ab}$ \\
\hline $5+4$ & $674,29 a$ & $12,63 a$ & $3,68 \mathrm{ab}$ & $21,42 a$ & $16,35 \mathrm{ab}$ & $19,88 \mathrm{ab}$ \\
\hline $10+0$ & $683,10 \mathrm{a}$ & $12,94 \mathrm{a}$ & $3,77 \mathrm{ab}$ & $22,55 a$ & $16,44 \mathrm{ab}$ & $19,30 \mathrm{ab}$ \\
\hline $10+2$ & $711,61 \mathrm{a}$ & $12,51 \mathrm{a}$ & $3,90 \mathrm{ab}$ & $21,96 a$ & $16,78 \mathrm{ab}$ & $19,98 \mathrm{ab}$ \\
\hline $10+4$ & $714,36 \mathrm{a}$ & $14,94 \mathrm{a}$ & $3,90 \mathrm{ab}$ & $21,86 \mathrm{a}$ & $16,44 \mathrm{ab}$ & $19,26 \mathrm{ab}$ \\
\hline $20+0$ & $713,19 a$ & $13,89 \mathrm{a}$ & $3,75 \mathrm{ab}$ & $22,20 \mathrm{a}$ & $16,32 \mathrm{ab}$ & $19,17 \mathrm{ab}$ \\
\hline $20+2$ & $750,90 a$ & $15,04 \mathrm{a}$ & $3,72 \mathrm{ab}$ & $22,29 a$ & $16,23 \mathrm{ab}$ & $19,28 \mathrm{ab}$ \\
\hline $20+4$ & $912,44 a$ & $19,08 \mathrm{a}$ & $4,09 \mathrm{a}$ & $22,54 \mathrm{a}$ & $16,95 \mathrm{a}$ & $18,70 \mathrm{~b}$ \\
\hline CV (\%) & 22,67 & 27,99 & 14,49 & 6,55 & 4,83 & 8,23 \\
\hline
\end{tabular}

Médias seguidas por letras distintas, dentro da mesma coluna, diferem entre si, pelo teste de Tukey, ao nível de 5\% de probabilidade. 
TABELA 3 - Influência do ácido giberélico e do thidiazuron sobre a massa fresca do cachos, do engaço e das bagas, comprimento e diâmetro das bagas e teor de sólidos solúveis totais da cv. BRS Clara. Jales, 2003.

\begin{tabular}{|c|c|c|c|c|c|c|}
\hline \multirow{3}{*}{$\begin{array}{c}\text { Trat amento } \\
\left(\mathrm{AG}_{3}+\mathrm{TDZ}^{2}\right) \\
\left(\mathrm{mg}^{-L^{-1}}\right)\end{array}$} & \multicolumn{3}{|c|}{ Massa Fresca } & \multirow[t]{2}{*}{ Comprimento Baga } & \multirow[t]{2}{*}{ Diâmetro Baga } & \multirow{3}{*}{$\begin{array}{c}\text { SST } \\
\left({ }^{0} \text { Brix }\right)\end{array}$} \\
\hline & Cacho & Engaço & Baga & & & \\
\hline & \multicolumn{3}{|c|}{ (g) } & \multicolumn{2}{|c|}{$(\mathrm{mm})$} & \\
\hline $0+0$ & $686,25 a$ & $13,39 a$ & $3,15 b$ & $20,60 \mathrm{~b}$ & $15,45 b$ & $20,22 a$ \\
\hline $5+5$ & $790,31 \mathrm{a}$ & $14,72 \mathrm{a}$ & $3,78 \mathrm{ab}$ & $22,47 \mathrm{ab}$ & $16,16 a b$ & $19,18 \mathrm{ab}$ \\
\hline $5+10$ & $735,88 \mathrm{a}$ & $13,67 \mathrm{a}$ & $3,86 a b$ & $21,92 \mathrm{ab}$ & $16,45 \mathrm{ab}$ & $20,07 \mathrm{a}$ \\
\hline $10+0$ & $683,10 \mathrm{a}$ & $12,82 \mathrm{a}$ & $3,77 \mathrm{ab}$ & $22,56 \mathrm{ab}$ & $16,44 \mathrm{ab}$ & $19,30 \mathrm{ab}$ \\
\hline $10+5$ & $957,54 \mathrm{a}$ & $19,55 \mathrm{a}$ & $4,37 \mathrm{a}$ & $22,63 \mathrm{ab}$ & $17,07 \mathrm{a}$ & $19,17 \mathrm{ab}$ \\
\hline $10+10$ & $727,71 \mathrm{a}$ & $15,92 \mathrm{a}$ & $3,79 \mathrm{ab}$ & $21,63 \mathrm{ab}$ & $16,27 \mathrm{ab}$ & $19,93 \mathrm{ab}$ \\
\hline $20+0$ & $713,19 a$ & $13,89 \mathrm{a}$ & $3,74 \mathrm{ab}$ & $22,20 \mathrm{ab}$ & $16,32 \mathrm{ab}$ & $19,17 \mathrm{ab}$ \\
\hline $20+5$ & $999,40 \mathrm{a}$ & $20,75 a$ & $4,10 \mathrm{ab}$ & $22,42 \mathrm{ab}$ & $16,96 a$ & $18,53 \mathrm{ab}$ \\
\hline $20+10$ & $977,05 \mathrm{a}$ & $21,55 \mathrm{a}$ & $4,56 \mathrm{a}$ & $23,31 \mathrm{a}$ & $17,44 \mathrm{a}$ & $17,18 b$ \\
\hline CV (\%) & 25,32 & 33,22 & 14,31 & 5,85 & 4,69 & 7,78 \\
\hline
\end{tabular}

Médias seguidas por letras distintas, dentro da mesma coluna, diferem entre si, pelo teste de Tukey, ao nível de 5\% de probabilidade.

TABELA 4 - Influência do ácido giberélico, aplicado antes e depois da floração, sobre a massa fresca do cachos, do engaço e das bagas, comprimento e diâmetro das bagas e teor de sólidos solúveis totais da cv. BRS Clara. Jales, 2003.

\begin{tabular}{|c|c|c|c|c|c|c|}
\hline \multirow{3}{*}{$\begin{array}{c}\text { Tratamento } \\
(\text { AF + DP) } \\
\left(\text { mg. }^{-1}\right)\end{array}$} & \multicolumn{3}{|c|}{ Massa Fresca } & \multirow[t]{2}{*}{ Comprimento Baga } & \multirow[t]{2}{*}{ Diâmetro Baga } & \multirow{3}{*}{$\begin{array}{c}\text { SST } \\
\left({ }^{0} \text { Brix }\right)\end{array}$} \\
\hline & Cacho & Engaço & Baga & & & \\
\hline & \multicolumn{3}{|c|}{ (g) } & \multicolumn{2}{|c|}{$(\mathrm{mm})$} & \\
\hline $0+0$ & $532,95 \mathrm{bc}$ & $10,27 \mathrm{ab}$ & $3,29 \mathrm{ab}$ & $21,26 b c$ & $15,43 a b c$ & $22,02 \mathrm{ab}$ \\
\hline $0+20$ & $767,27 \mathrm{a}$ & $14,44 \mathrm{a}$ & $3,79 \mathrm{a}$ & $22,40 \mathrm{abc}$ & $16,25 \mathrm{a}$ & $20,00 \mathrm{c}$ \\
\hline $0+40$ & $616,16 \mathrm{ab}$ & $12,12 \mathrm{ab}$ & $3,92 \mathrm{a}$ & $23,34 \mathrm{ab}$ & $16,35 \mathrm{a}$ & $20,28 b c$ \\
\hline $20+0$ & $290,23 d$ & $7,51 \mathrm{~b}$ & $3,37 \mathrm{ab}$ & $21,70 \mathrm{abc}$ & $15,32 \mathrm{abc}$ & $21,58 \mathrm{abc}$ \\
\hline $20+20$ & $438,16 \mathrm{bcd}$ & $9,95 \mathrm{ab}$ & $3,65 \mathrm{a}$ & $22,79 \mathrm{abc}$ & $15,64 \mathrm{ab}$ & $22,62 \mathrm{a}$ \\
\hline $20+40$ & $292,15 d$ & $7,51 \mathrm{~b}$ & $3,77 \mathrm{a}$ & $23,43 \mathrm{ab}$ & $15,89 \mathrm{a}$ & $23,17 \mathrm{a}$ \\
\hline $40+0$ & $239,26 \mathrm{~d}$ & $7,85 b$ & $2,88 \mathrm{~b}$ & $20,93 \mathrm{c}$ & $14,50 \mathrm{c}$ & $23,37 \mathrm{a}$ \\
\hline $40+20$ & $342,12 \mathrm{~cd}$ & $10,19 \mathrm{ab}$ & $3,48 \mathrm{ab}$ & $23,18 \mathrm{abc}$ & $14,80 \mathrm{bc}$ & $22,85 \mathrm{a}$ \\
\hline $40+40$ & $393,46 \mathrm{bcd}$ & $9,95 \mathrm{ab}$ & $3,74 a$ & $23,55 \mathrm{a}$ & $15,57 \mathrm{ab}$ & $23,07 \mathrm{a}$ \\
\hline CV (\%) & 26,66 & 24,92 & 10,06 & 5,33 & 3,63 & 4,32 \\
\hline
\end{tabular}

*AF - 6 dias antes do início da floração e DP - 07 dias depois da plena floração.

Médias seguidas por letras distintas, dentro da mesma coluna, diferem entre si, pelo teste de Tukey, ao nível de 5\% de probabilidade.

Com relação à massa fresca do engaço, não houve diferenças estatísticas significativas entre a maioria dos tratamentos, apesar do aumento da massa do engaço com o aumento das concentrações dos reguladores de crescimento. Embora tenham sido testadas diversas concentrações do $\mathrm{AG}_{3}, \mathrm{CPPU}$ e TDZ, não houve aumento da espessura do engaço de modo a causar prejuízos aos cachos, o que também foi constatado por Cooper et al. (1993), na cv. Thompson Seedless. Segundo esses autores, o incremento das doses de ácido giberélico aumenta também a incidência de degrana no período pós-colheita devido à perda de flexibilidade dos pedicelos. Pérez \& Morales (1999) relacionaram o aumento da espessura do engaço com o incremento das doses de ácido giberélico, ao aumento da atividade da enzima peroxidase solúvel.

Quanto à massa fresca e ao comprimento das bagas, observouse um aumento com a aplicação dos reguladores de crescimento, principalmente nas maiores concentrações, porém sem diferenças estatísticas significativas para a maioria dos tratamentos (Tabelas 1 a 3). No caso das aplicações do $\mathrm{AG}_{3}$ antes e depois da floração (Tabela 4), pode-se verificar que os maiores incrementos nessas variáveis ocorrem quando as aplicações foram realizadas após a floração, o que, segundo Pires \& Botelho (2001), pode ter sido devido à hidrólise do amido resultante da produção da $\alpha$-amilase, o que aumenta a pressão osmótica do suco celular, fazendo com que ocorra a entrada de água e, conseqüentemente, favoreça a expansão da célula.

Com relação ao diâmetro, que é a variável mais importante em se tratando de uva sem semente, já que normalmente é exigido um diâmetro maior do que $16-17 \mathrm{~mm}$ para exportação, houve aumento de cerca de $1,7 \mathrm{~mm}$ nas bagas que foram tratadas com as concentrações mais elevadas dos reguladores de crescimento (60mg.L $\mathrm{L}^{-1} \mathrm{de} \mathrm{AG}_{3}$ (Tabela 1), 20mg.L. $\mathrm{L}^{-1}$ de $\mathrm{AG}_{3}+4 \mathrm{mg} . \mathrm{L}^{-1}$ de CPPU (Tabela 2) e $10 \mathrm{mg} . \mathrm{L}^{-1} \mathrm{de} \mathrm{AG}_{3}+$ $5 \mathrm{mg} . \mathrm{L}^{-1}$ de TDZ (Tabela 3 ). O efeito sinérgico da utilização de citocininas
(TDZ ou CPPU) em conjunto com o $\mathrm{AG}_{3}$ observado neste trabalho também é mencionado por Reynolds et al. (1992), que trabalharam com quatro seleções de uvas de mesa sem sementes.

No caso dos tratamentos utilizando somente o $\mathrm{AG}_{3}$ (Tabela 1), não foram observadas diferenças estatísticas significativas entre os tratamentos, embora tenha ocorrido um pequeno incremento do diâmetro nas maiores concentrações. Devido a este fato, optou-se pela recomendação da concentração de $60 \mathrm{mg} \cdot \mathrm{L}^{-1} \mathrm{de}^{\mathrm{A}} \mathrm{G}_{3}$, uma vez que foi a concentração mais baixa e em dose única que proporcionou diâmetro de baga superior aos $16 \mathrm{~mm}$. A utilização de concentrações mais baixas também é válida para o uso combinado dos reguladores, desde que não exista diferença estatística significativa entre as concentrações. Aplicações do $\mathrm{AG}_{3}$ antes da floração (Tabela 4) também não foram eficientes para aumento do diâmetro das bagas.

Quanto aos teores de SST das bagas, observou-se redução de 3 a $5^{\circ}$ Brix com a utilização dos reguladores de crescimento em relação aos tratamentos sem aplicação (Tabelas 1 a 3). Entretanto, os valores observados são bastante superiores ao valor mínimo exigido para a comercialização de uvas finas de mesa (14 Brix) (Almeida, 2003). Essa redução no teor de SST pode provocar aumento no ciclo de cerca de uma a duas semanas, o que foi observado por Ben Tal (1990), na cv. Thompson Seedless, e por Leão et al. (1999), que trabalharam com a cv. Perlette.

O aumento nos teores de SST, observado na Tabela 4, pode ser atribuído ao abortamento de flores causado pelas aplicações do $\mathrm{AG}_{3}$ antes da floração, o que reduziu o número de bagas nos cachos, permitindo o maior acúmulo de açúcares nas mesmas.

Fazendo-se uma análise dos experimentos em conjunto, podese dizer que a cv. BRS Clara apresenta pouca resposta à aplicação de reguladores de crescimento. Entretanto, é possível a obtenção de bagas 
com diâmetro médio em torno de 16-17mm, que é o mínimo exigido para exportação. Com relação ao diâmetro, é importante salientar que, durante a condução dos experimentos, não foram efetuados desbastes com tesouras, nos quais são eliminadas as bagas menores. Essa operação, que é feita normalmente nos pomares comerciais, aumenta o diâmetro médio das bagas, não só pela eliminação das menores, mas também pela redução do número de bagas remanescentes no cacho.

Com base nos resultados obtidos, pode-se concluir que a utilização do $\mathrm{AG}_{3}$, isolado ou em conjunto com o CPPU ou TDZ, melhora as características do cacho da cv. BRS Clara; o uso do TDZ e do CPPU em conjunto com $\mathrm{o} \mathrm{AG}_{3}$ produz efeito sinérgico, proporcionando melhor resposta do que o uso isolado do $\mathrm{AG}_{3}$; os tratamentos com $60 \mathrm{mg}$. $\mathrm{L}^{-1} \mathrm{de}$ $\mathrm{AG}_{3}, 20 \mathrm{mg} . \mathrm{L}^{-1}$ de $\mathrm{AG}_{3}+4 \mathrm{mg} . \mathrm{L}^{-1}$ de CPPU e $10 \mathrm{mg} . \mathrm{L}^{-1}$ de $\mathrm{AG}_{3}+5 \mathrm{mg} . \mathrm{L}^{-1} \mathrm{de}$ TDZ proporcionam os melhores resultados para aumento no diâmetro das bagas; a aplicação de $\mathrm{AG}_{3}$ antes da floração da cv. BRS Clara provoca abortamento excessivo, reduzindo a qualidade comercial dos cachos; a aplicação de concentrações elevadas de $\mathrm{AG}_{3}, \mathrm{CPPU}$ e TDZ provoca redução nos teores de SST da cv. BRS Clara.

\section{REFERÊNCIAS}

ALMEIDA, G.V.B. Mercado interno: a uva no contexto do mercado de frutas. In: CONGRESSO BRASILEIRO DE VITICULTURA E ENOLOGIA, 10., 2003, Bento Gonçalves. Anais... Bento Gonçalves: Embrapa Uva e Vinho, 2003. p.161-165.

BEN TAL, Y. Effects of gibberellin treatments on ripening and berry drop from Thompson Seedless grapes. American Journal of Enology and Viticulture, Daves, v.41, n.2, p.142-146, 1990.

CAMARGO, U.A. Melhoramento genético: variedades de uvas sem sementes para o Brasil. In: CONGRESSO BRASILEIRO DE VITICULTURA E ENOLOGIA, 10., 2003, Bento Gonçalves. Anais... Bento Gonçalves: Embrapa Uva e Vinho, 2003. p.171-172.

CAMARGO, U.A.; MASHIMA, C.H.; CZERMAINSKI, A. B. C. Avaliação de cultivares de uvas apirênicas no Vale do São Francisco. Bento Gonçalves: Embrapa Uva e Vinho, 1997. 8p. (Comunicado Técnico, 26).
CAMARGO, U.A.; NACHTIGAL, J.C.; MAIA, J.D.G; OLIVEIRA, P.R.D.; PROTAS, J.F. da S. BRS Clara: nova cultivar de uva branca de mesa sem semente. Bento Gonçalves: Embrapa Uva e Vinho, 2003. $4 p$. (Comunicado Técnico, 46).

COOPER, T.; REMATALES, J.; BANGERTH, F. Berry drop occurence as affected by $\mathrm{GA}_{3}$ and promalin applications in Thompson Seedless grapes. Acta Horticulturae, Wageningen, v.329, p.134-136, 1993.

LEÃO, P.C.S. Comportamento das variedades de uva sem sementes Crimson Seedless e Fantasy Seedless no Submédio São Francisco. Petrolina-PE: Embrapa Semi-Árido, 2001. 18p. (Boletim de Pesquisa e Desenvolvimento, 56).

LEÃO, P.C.S.; LINO JUNIOR, E.C.; SANTOS, E.S. Efeitos do CPPU e do ácido giberélico sobre o tamanho de bagas de uvas Perlette cultivadas no Vale do São Francisco. Revista Brasileira de Fruticultura, Cruz das Almas, v.21, n.1, p.74-78, 1999.

NACHTIGAL, J.C. Avanços tecnológicos na produção de uvas de mesa. In: CONGRESSO BRASILEIRO DE VITICULTURAE ENOLOGIA, 10., 2003, Bento Gonçalves. Anais... Bento Gonçalves: Embrapa Uva e Vinho, 2003. p.167-170.

PÉREZ, F.J.; MORALES, V. A basic peroxidase isoenzyme from the grape pedicel is induced by gibberellic acid. Australian Journal of Plant Physiology, Collingwood, v.26, p.387-390, 1999.

PIRES, E.J.P.; BOTELHO, R. V. Emprego de reguladores de crescimento em viticultura. In: SIMPÓSIO MINEIRO DE VITICULTURA E ENOLOGIA, 1., 2002, Andradas. Anais... Caldas: Epamig, 2002.p.5981.

PIRES, E.J.P.; BOTELHO, R.V. Uso de reguladores vegetais na cultura da videira. In: SIMPÓSIO BRASILEIRO SOBRE UVAS DE MESA, 1., 2000, Ilha Solteira-SP. Anais.... p.129-147.

PROTAS, J.F. da S.; CAMARGO, U.A.; MELO, L.M.R. de. A viticultura brasileira: realidade e perspectivas. In: SIMPÓSIO MINEIRO DE VITICULTURA E ENOLOGIA, 1., 2002, Andradas. Anais... Caldas: Epamig, 2002.p.11-32.

REYNOLDS, A.G.; WARDLE, D.A.; ZUROWSKI, C.; LOONEY, N.E. Phenylureas CPPU and thidiazuron effect yield components, fruit composition, and storage potential of four seedless grape selections. Journal of the American Society for Horticultural Science, Alexandria, v.117, n.1, p.85-89, 1992. 